EXTENDED REPORT

\title{
p53 Expression in rheumatoid and psoriatic arthritis synovial tissue and association with joint damage
}

\author{
G Salvador, R Sanmarti, A Garcia-Peiró, J R Rodríguez-Cros, J Muñoz-Gómez, J D Cañete
}

Ann Rheum Dis 2005;64:183-187. doi: 10.1136/ard.2004.024430

See end of article for authors' affiliations

....................

Correspondence to: Dr J D Cañete, Unitat d'Artritis, Servei de Reumatologia, Hospital Clínic and Institut dlnvestigacions Biomèdiques August $\mathrm{Pi} i$ Sunyer (IDIBAPS), Villarroel 170, 08036 Barcelona, Spain; jcanete@clinic.ub.es

Accepted 22 August 2004
Background: Overexpression and functional mutations of p53 have been found in the synovial tissue (ST) of patients with rheumatoid arthritis (RA), but their clinical significance remains unclear.

Objective: To analyse p53 expression in the ST of patients with RA and psoriatic arthritis (PsA) and its association with joint damage.

Methods: Synovial biopsy specimens were obtained by arthroscopy in 45 patients (27 RA, 18 PsA). Radiographs of hands, feet, and the joint undergoing arthroscopy were obtained to evaluate the presence of erosive disease. Synovial cell populations were analysed using CD4, CD8, CD138, CD20, and CD68 monoclonal antibodies (mAbs). The $\mathrm{p} 53$ protein was determined by immunohistology using DO7 mAb in 34 patients (18 RA, 16 PsA). In 11 patients with early RA, the association between p53 and 1 year progression of radiographic damage was analysed using the Larsen-Scott method.

Results: The p53 protein was detected in 16/18 (89\%) patients with RA and in 9/16 (56\%) patients with PsA, but its expression in RA was significantly higher than in PsA. In RA, p53 expression was significantly associated with erosive disease, and its scores were higher in patients with radiological progression. CD68 expression was also associated with erosions and radiological progression in RA. No association was found between either p53 or CD68 and erosive disease in PsA.

Conclusions: These results suggest that p53 ST overexpression and association with joint damage is characteristic of RA rather than PsA, and that p53 ST expression might be a prognostic marker of joint damage in RA.
$\mathrm{O}$ verexpression and functional mutations of the tumour suppressor p53 protein have been demonstrated in rheumatoid arthritis (RA) synovial tissue, most extensively in patients with advanced, destructive disease. ${ }^{1}$ Although overexpression of p53 in tissues was originally thought to be a surrogate marker for mutations, ${ }^{2}$ subsequent studies have demonstrated wild-type p53 expression in RA and other inflammatory diseases, including reactive arthritis and inflammatory osteoarthritis. ${ }^{3}$ Recent studies suggest that p53 induction is a general phenomenon in inflammation, directed at modulating normal inflammatory responses. ${ }^{4}$ Although a higher p53 expression has been demonstrated in synovial tissue (ST) from patients with destructive RA disease, no prospective studies exploring the association between p53 expression and joint damage have been published.

Psoriatic arthritis (PsA) develops in about $5-25 \%$ of patients with psoriasis and may be as severe as RA, with almost $20 \%$ of patients developing severe, destructive, deforming arthritis. ${ }^{5}$ Although classified as a spondyloarthropathy, PsA shares pathogenic mechanisms with RA, including the Thl cytokine derived pattern, ${ }^{6}$ ST expression of angiogenic inductors, ${ }^{7}$ the central role of the proinflammatory cytokine tumour necrosis factor $\alpha$ in its pathogenesis, ${ }^{8}$ and RANKL derived mechanisms of bone erosion. ${ }^{9}$ Although p53 overexpression has been recorded in the cutaneous lesions of psoriasis, ${ }^{10}$ studies focusing on $\mathrm{p} 53$ expression in the ST of patients with PsA are lacking.

This study aimed at analysing p53 protein expression and different cell markers in ST from patients with RA and PsA and its association with radiographic damage.

\section{PATIENTS AND METHODS}

Patients

Forty five consecutive patients (24F/21M) with active arthritis attending the outpatient clinic of the rheumatology department of the Hospital Clinic of Barcelona were included. Twenty seven fulfilled the American College of Rheumatology criteria for RA and 18 fulfilled the Moll and Wright criteria for PsA. Laboratory tests included serum C reactive protein levels (CRP), erythrocyte sedimentation rate (ESR), rheumatoid factor (RF; by nephelometry), and HLAB27 status (by polymerase chain reaction). All patients underwent diagnostic or therapeutic arthroscopy of one affected joint after giving informed consent. Radiographs of hands, feet, and the joint undergoing arthroscopy were obtained in all cases at inclusion in the study. Hands and feet radiographs were obtained after 12 months of follow up in a subgroup of patients with RA with less than 12 months of disease duration. The study was approved by the ethics committee of the Hospital Clinic.

\section{Arthroscopy and synovial biopsies}

Arthroscopies were performed in a sterile room, with local anaesthesia and without knowledge of the radiographic status of the joint undergoing arthroscopy. We used a $2.7 \mathrm{~mm}$ arthroscope for knees, wrists, and elbows and one of $1.9 \mathrm{~mm}$ for metacarpophalangeal joints (Storz, Tuttlingen, Germany). ${ }^{11}$ Between six and eight biopsy samples were taken from the affected synovium. Synovial samples were fixed in $10 \%$ neutral buffered formalin, dehydrated, and then embedded in paraffin using an automated tissue processor.

\section{Immunohistological analysis}

p53

The immunohistological method used to determine p53 expression has been previously described. ${ }^{3}$ Briefly, paraffin

Abbreviations: CRP, C reactive protein; $E S R$, erythrocyte sedimentation rate; $\mathrm{mAb}$, monoclonal antibody; $\mathrm{PsA}$, psoriatic arthritis; RA, rheumatoid arthritis; RF, rheumatoid factor; ST, synovial tissue 
Table 1 Clinical and serological characteristics of patients with RA and PsA

\begin{tabular}{lll}
\hline & $\begin{array}{l}\text { Rheumatoid } \\
\text { arthritis } \\
\text { (n= 27) }\end{array}$ & $\begin{array}{l}\text { Psoriatic arthritis } \\
\text { (n= 18) }\end{array}$ \\
\hline Women, No (\%) & $16(59)$ & $8(44)$ \\
Age (years), mean (SD) & $54.1(15.6)$ & $44.9(13.1)$ \\
Disease duration (months), & $60.3(90)$ & $88.6(82.1)$ \\
mean (SD) & $19(70)$ & $0(0)$ \\
RF+, No (\%) & $2(7)$ & $1(6)$ \\
HLA-B27 +, No (\%) & $12(44)$ & $6(33)$ \\
Baseline erosions*, No (\%) & $43.7(27.5)$ \\
ESR (mm/1st h), mean (SD) & $35.8(31.2)$ & $26(29)$ \\
CRP (mg/l), mean (SD) & $23(35)$ & 26 \\
\hline
\end{tabular}

*Number of patients with radiological erosions at the time of arthroscopy.

sections of 4-6 $\mu \mathrm{m}$ were immunostained with mouse anti-p53 monoclonal antibody (mAb) DO7 (Novocastra Laboratories, Newcastle, UK) diluted to a final concentration of $0.2 \mu \mathrm{g} / \mathrm{ml}$. As secondary antibodies, donkey antimouse antibodies labelled with biotin (Jackson Immuno-Research, West Grove, PA) were diluted to a final concentration of $5 \mu \mathrm{g} / \mathrm{ml}$ and incubated for 30 minutes. Incubation with SA-horseradish peroxidase for 30 minutes was followed by incubation with biotinylated tyramide (TSA Biotin System, Perkin-Elmer, Boston, MA).

As previous studies carried out this amplification method using frozen preparations, a confirmatory test for optimal p53 detection in paraffin sections using cyanine 3-fluorophore labelled tyramides (TSA Fluorescence System, Perkin-Elmer), a method in which we have experience, was performed as previously. ${ }^{12}$ Colon adenocarcinoma tissue preparations served as a positive $\mathrm{p} 53$ control. In each procedure, additional sections were incubated with only the secondary antibody and detection system to exclude non-specific binding.

\section{Cellular infiltrate}

Staining of serial sections was performed using the following mAbs: anti-CD4 (1F6; Novocastra), anti-CD8 (4b11; Novocastra), anti-C20 (L26; Dako), anti-CD68 (KP-1; Dako), and anti-CD138 (B-B4; Santacruz, San Diego, CA), as previously described. ${ }^{7}$

\section{Microscopic analysis}

The p53 protein was detected and scored in the lining, sublining, and endothelium of ST. A p53 total score was also obtained. All sections were randomly evaluated by two independent observers who were unaware of the clinical data. The compensated $\kappa$ value of interobserver reliability was 0.85 . Immunohistochemical results for p53 expression were scored as previously reported. ${ }^{3}$

Expression of the different cell markers (CD4, CD8, CD20, CD68, and CD138) was scored semiquantitatively. ${ }^{7}$ Scores were individualised for each marker.

\section{Radiological analysis}

At study entry, radiographs of hands, feet, and the joint undergoing arthroscopy were obtained in all cases, in order to classify patients' disease as erosive or non-erosive. Furthermore, hands and feet radiographs were made after 1 year of follow up in 11/18 patients with RA. This subgroup of patients was included because their disease duration was $<12$ months (mean (SD) 6.4 (3.5) months). The remaining seven patients had a disease duration $>36$ months (mean (SD) 146 (97.6) months). A change of two or more units using the Larsen-Scott method was used to define 1 year radiographic progression in these patients. ${ }^{13} 14$ All radiographs were scored by the same observer chronologically.

\section{Statistical analysis}

Data were analysed using the SPSS 10.0 statistical software package, under licence to the Hospital Clinic of Barcelona. The univariate analysis was performed using the $\chi^{2}$ test, Student $t$ test, or the non-parametric Mann-Whitney test. The non-parametric Spearman correlation between biological parameters (CRP and ESR) and the p53 protein was calculated.

Risk association, adjusted for CRP and ESR, between the p53 protein and the presence of RA was estimated by multivariate logistic regression. The level of statistical significance was established at $\mathrm{p} \leqslant 0.05$.

\section{RESULTS}

\section{Clinical features}

Forty five patients: 27 RA (16F/11M, 19 (70\%) RF+, mean (SD) age 54.1 (15.6) years, mean disease duration 60.3 (90) months) and 18 PsA (8F/10M, mean (SD) age 44.9 (13.1) years, mean disease duration 88.6 (82.1) months), 11 with RF negative polyarthritis and seven with oligoarthritis, were included in the study. There were no significant differences in disease duration between the two groups of patients. Arthroscopy was performed in the knee $(\mathrm{n}=35)$, wrist $(\mathrm{n}=8)$, elbow $(\mathrm{n}=1)$, and metacarpophalangeal joints $(\mathrm{n}=1)$. At inclusion, most patients were treated with nonsteroidal anti-inflammatory drugs and low doses of prednisone (mean daily dose $5 \mathrm{mg}$ ). Fifteen patients in the RA group also received disease modifying antirheumatic drugs
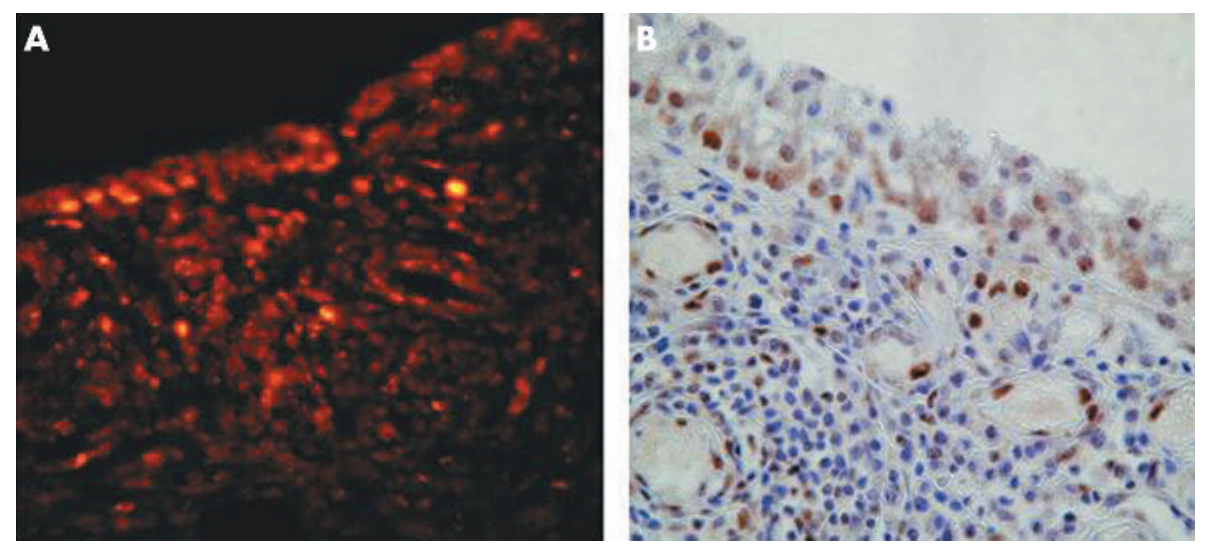

Figure 1 Immunofluorescence (A) and immunohistochemical (B) staining for p53 in ST of the same patient with RA. Technique with tyramide enhancement (see text). Original magnification $\times 600$ (A and B). 

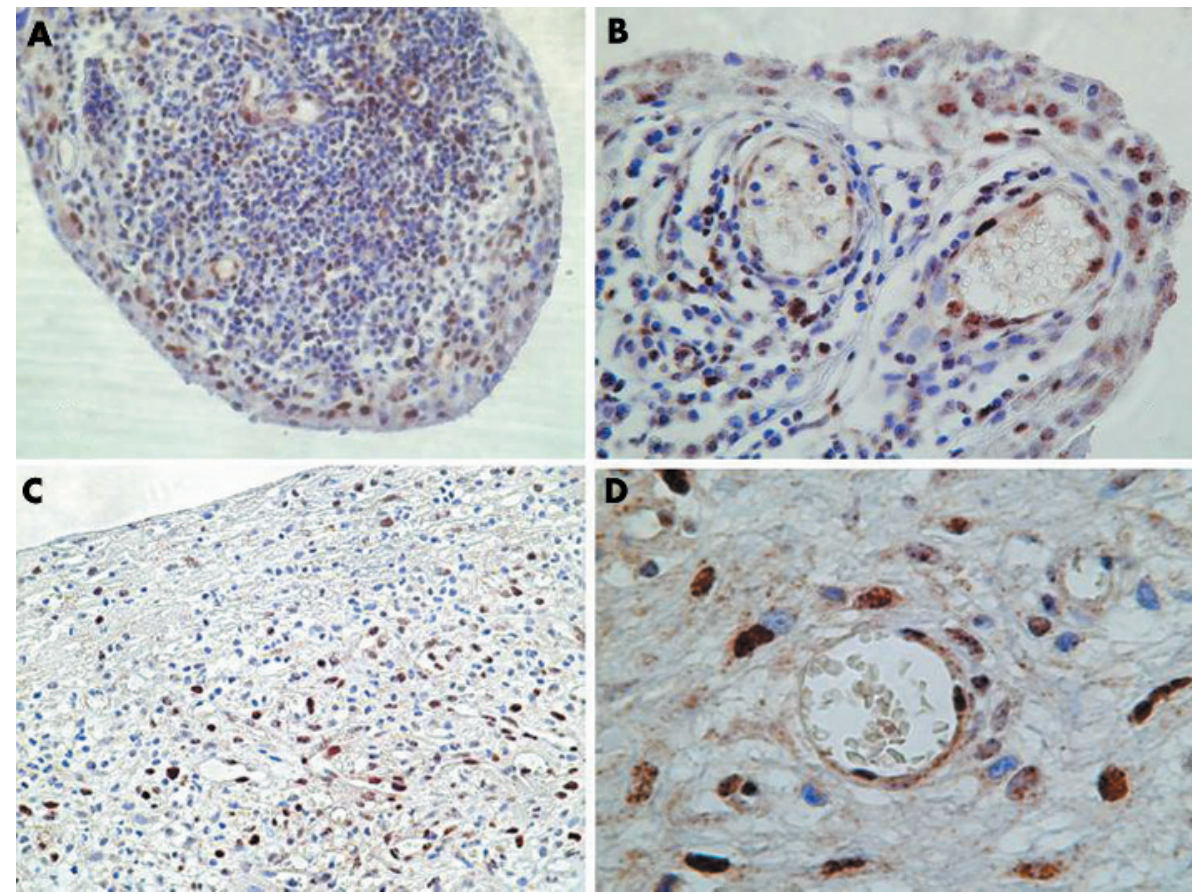

Figure 2 Immunostaining for p53 tissue in ST from patients with RA (A and B) and PsA (C and D). Original magnification $\times 400(A) ; \times 600(B) ; \times 400$ (C), and $\times 1000$ (D).

(9 $(60 \%)$ intramuscular gold salts, and $6(40 \%)$ methotrexate) and 12 patients in the PsA group were treated with methotrexate. Table 1 shows the demographic and clinical characteristics of the patients.

\section{Radiological data}

Eighteen (12 RA/6 PsA) of 45 (40\%) patients had radiological erosions at the moment of arthroscopy. As expected, the percentage of erosive disease in RA was significantly greater in patients with established disease than in those with RA of $<12$ months: $87.5 \% \vee 26 \%$. No significant differences in biological parameters of inflammation (CRP or ESR) were seen between patients with and without erosions (table 1). Radiographic progression was found in 4/11 patients with RA in whom 1 year radiological progression was analysed. At the end of follow up, all these patients were treated with disease modifying antirheumatic drugs: four with methotrexate, six with parenteral gold, and one with hydroxychloroquine.

\section{Immunohistolology of p53}

The final p53 protein immunohistochemical analysis included 34 (18 RA, 16 PsA) of 45 patients; 11 patients were excluded because their tissue preparations, principally

Table 2 p53 expression* in different areas of synovial tissue of patients with RA and PsA

\begin{tabular}{llll}
\hline & $\begin{array}{l}\text { Rheumatoid } \\
\text { arthritis } \\
(\mathbf{n}=18)\end{array}$ & $\begin{array}{l}\text { Psoriatic arthritis } \\
\text { (n= 16) }\end{array}$ & p Value \\
\hline Global p53 & $2.0(1.4)$ & $0.8(0.9)$ & 0.008 \\
Endothelial p53 & $2.9(1.3)$ & $1.6(1.5)$ & 0.020 \\
Lining p53 & $3.0(1.0)$ & $0.9(1.2)$ & $<0.0001$ \\
Sublining p53 & $2.0(1.3)$ & $0.8(1.0)$ & 0.012 \\
ESR (mm/1st h) & $38.9(35.2)$ & $43.7(27.5)$ & 0.27 \\
CRP (mg/l) & $26(40)$ & $26(28)$ & 0.78 \\
\hline
\end{tabular}

Results are shown as mean (SD).

*p53 was only scored in $34 / 45$ patients.
Table 3 Clinical and radiological characteristics of patients according to $\mathrm{p} 53$ scores

\begin{tabular}{|c|c|c|}
\hline & $\begin{array}{l}p 53 \geqslant 2 \\
(n=13)\end{array}$ & $\begin{array}{l}p 53 \leqslant 1 \\
(n=21)\end{array}$ \\
\hline $\operatorname{Sex}(F)$, No $(\%)$ & $8(62)$ & $12(57)$ \\
\hline Age, mean (SD) & $55.1(16.4)$ & 47.1 (13.3) \\
\hline $\begin{array}{l}\text { Mean evolution of disease (months), } \\
\text { mean (SD) }\end{array}$ & 75.1 (77) & $83.8(99.3)$ \\
\hline Baseline erosions, No (\%) & $8(62)$ & $5(24)$ \\
\hline Diagnostic & $10 R A / 3 P_{s} A$ & $8 \mathrm{RA} / 13 \mathrm{PsA}_{\mathrm{s}}$ \\
\hline
\end{tabular}

obtained from wrist and metacarpophalangeal joints, did not include sufficient synovial membrane for adequate p53 evaluation. The p53 immunofluorescence staining and peroxidase staining were concordant and positive in 16/18 patients with RA and in 9/16 patients with PsA (fig 1). Staining was nuclear and cytoplasmic (fig 2), but only p53 protein stained with the peroxidase technique was scored.

\section{p53 expression in RA and PsA}

The mean (SD) scores for total p53 expression were significantly higher in patients with RA than in patients with PsA (2.0 (1.4) $v 0.8$ (0.9); $\mathrm{p}=0.008)$. The highest scores, 2,3 , and 4 ( $>5 \%$ of positive cells), were found in 10/18 (56\%) patients with RA compared with $3 / 16(19 \%)$ patients with PsA. A score of 4 was found in 4/18 (22\%) patients with RA, but in no patient with PsA. Conversely, a p53 total score of 0 was found in $2 / 18(11 \%)$ patients with RA compared with 7/16 (44\%) patients with PsA.

The scores for p53 expression in the lining, sublining, and endothelium were all also significantly higher in patients with RA than in patients with PsA (table 2).

The presence of p53 expression scores $\geqslant 2$ in all the areas analysed, adjusted by inflammation parameters (CRP and ESR), conferred an increased risk of having RA, with an odds ratio $=8.2(95 \%$ confidence interval 1.4 to $49.3 ; p=0.022)$ for 
Table 4 Expression of different cell markers in synovial tissue of patients with RA and PsA.

\begin{tabular}{llll}
\hline & $\begin{array}{l}\text { Rheumatoid arthritis } \\
(\mathbf{n}=\mathbf{2 7})\end{array}$ & $\begin{array}{l}\text { Psoriatic arthritis } \\
(\mathbf{n}=18)\end{array}$ & $\mathbf{p}$ Value \\
\hline CD4 & $2.9(0.7)$ & $2.1(1.0)$ & 0.012 \\
CD8 & $2.5(1.0)$ & $2.2(0.9)$ & 0.459 \\
CD20 & $2.0(1.4)$ & $1.5(1.3)$ & 0.235 \\
CD138 & $1.5(1.4)$ & $1.4(1.1)$ & 0.971 \\
CD68 & $2.0(1.3)$ & $1.7(0.96)$ & 0.117
\end{tabular}

Results are shown as mean (SD).

p53 total expression. The highest risk was with lining p53 expression (odds ratio $=31.5$ (95\% confidence interval 4.5 to $220.2 ; \mathrm{p}=0.001)$.

\section{p53 and radiographic damage}

Total and sublining p53 expression was associated with erosive disease $(p=0.012$ and $p=0.032$, respectively). However, this association was only present in the RA group: the scores of the p53 total, lining, sublining, and endothelial expression were significantly higher in patients with erosive RA than in those with non-erosive disease. Furthermore, none of the patients with erosive RA had a score of 0 , and 60$100 \%$ of patients with erosive RA had scores of 3 or 4 in the synovial areas analysed. Table 3 shows the clinical and radiological characteristics of the 34 patients according to p53 scores.

When analysing the 11 patients with RA in whom 1 year radiographic follow up was available, p53 synovial expression score was 4 in $3 / 4(75 \%)$ patients with progression, whereas $6 / 7(86 \%)$ patients without progression scored $\leqslant 1$. In all three patients with significant progression after l year (Larsen >10 units), p53 expression score was the maximum (4).

No association was found between p53 expression and erosive PsA, and the distribution of score 0 or scores 3-4 was similar in patients with erosive and non-erosive PsA.

\section{Immunohistology of cellular markers}

Only the mean score for T CD4+ lymphocytes was significantly higher in patients with RA than in those with PsA $(\mathrm{p}=0.012)$ (table 4$)$. The mean (SD) CD68+ cell expression score was significantly higher in patients with baseline erosions than in those without erosions (2.6 (1.1) $v$ 1.8 (1.1); $\mathrm{p}=0.03)$. However, this association was only valid for the RA group: erosive RA had significantly higher CD68+ scores than patients with non-erosive RA (3.1 ( 1$) v 1.9$ (1.3); p=0.03). Furthermore, $73 \%$ of patients with erosive RA had CD68+ scores of 3-4 compared with only $23 \%$ of patients with nonerosive RA. CD68+ expression mean score in the four patients with 1 year radiological progression was 3 , whereas in the seven patients without radiological progression the score was 1.7 .

\section{DISCUSSION}

In this study p53 was detected in almost all patients with RA in the synovial lining, sublining, and endothelium. We found an association between the mean p53 score and erosive disease at baseline in patients with RA. In addition, around $80 \%$ of patients with RA with erosion had the maximum p53 scores. Radiological progression after 1 year in RA was also associated with the highest p53 scores at study entry. These results confirm previous studies on p53 expression in the ST of patients with $\mathrm{RA}^{1{ }^{3}}$ and show, for the first time, a consistent association between p53 expression and joint damage. Other studies have not found significant p53 expression in RA ST, probably because less sensitive methods were used..$^{15} 16$

On the other hand, this study found that p53 is also expressed in PsA ST, although at a significantly lower level than in RA, and that this expression is not associated with joint damage. Only one previous study, published in abstract form, reported p53 expression in the nuclei of lining cells of six patients with PsA, although it provided no methodological, clinical, or p53 scoring data to compare with our study. ${ }^{17}$

The differential expression of p53 in RA and PsA in this study did not seem to be related to differences in disease duration or inflammatory activity, two factors that might affect p53 expression. ${ }^{4}$ This difference was maintained when each area of ST scored for p53 expression was compared between RA and PsA.

Our results suggest that p53 expression has distinct pathogenic consequences in RA compared with PsA because an association with joint damage was found only in RA, both at baseline and after 1 year of follow up. However, given the small number of patients prospectively analysed, this point needs to be confirmed in future studies. Another limitation of this study is that our results are principally based on knee synovium, because samples obtained from the wrist and metacarpophalangeal joint were insufficient to obtain a complete p53 score. Similar studies based on biopsy specimens obtained from small joints might be interesting in order to extend our results.

Although there are studies on p53 mutations and protein expression in RA and other inflammatory disorders, ${ }^{1-3}{ }^{18-20}$ and much fine basic research has been done on the topic, ${ }^{4}{ }^{21-23}$ the clinical significance of p53 expression in RA remains unclear. Originally, overexpression of p53 in tissues was thought to be a surrogate marker for mutation. The demonstration of p53 single nucleotide mutations in the synovium of patients with RA, predominantly in the lining layer, suggested that the oxidative environment of rheumatoid synovium induced these mutations. ${ }^{2}{ }^{18}$ Subsequently, the same authors also reported high p53 expression in the sublining. ${ }^{3}$ Muted p53 proteins have a longer half life than wild ones, which increases their expression. Furthermore, muted p53 might lose its growth control regulatory properties and might even induce synthesis of metalloproteinases and proinflammatory cytokines, causing joint damage. ${ }^{24}$ However, recent studies have found wild-type p53 in human inflammatory diseases, including $\mathrm{RA}$, reactive arthritis, and psoriasis, ${ }^{20}$ and this has been confirmed in animal models of arthritis. ${ }^{4}$ One explanation for these findings would be that total p53 expression in RA includes muted and wild forms, with muted p53 predominating in the lining and leading to a net destructive effect. On the other hand, p53 expression in other inflammatory rheumatic diseases, including PsA, might be wild-type, with a protective role. However, this important question should be examined by specific p53 mutation studies.

A recent study using synovial RA fibroblasts and the noninflammatory SCID mouse co-implantation model of RA, suggests that $\mathrm{p} 53$ at sites of cartilage invasion could be induced during the destructive process driven by activated RA synovial fibroblasts, without the participation of inflammatory cells. ${ }^{23}$ Although obtained in an animal model, these results agree with the finding that $\mathrm{p} 53$ is overexpressed in the lining of RA, the site of previously demonstrated mutations, and that its expression correlates with joint damage.

Although PsA may share several pathogenic mechanisms with RA, ${ }^{6-9}$ our study suggests that differential pathogenic mechanisms of joint damage exist between RA and PsA. In PsA, erosions occur less commonly than in RA and progression to joint destruction occurs at a slower rate. ${ }^{25}$ Some authors have suggested that synovitis in PsA might be a 
secondary phenomenon to enthesitis or osteitis, ${ }^{26}$ or that synovitis may not be responsible for the destructive lesions characteristic of PsA. ${ }^{27}$ In addition, it has also been reported that angiogenesis plays a fundamental part in the pathophysiology of synovitis in PsA as compared with RA, ${ }^{28}$ and that the vascular synovial morphology is distinct in PsA and RA. ${ }^{11}{ }^{29}$ However, in a recent study, the different degrees of radiological progression of PsA and RA were not explained by a difference in the expression of matrix metalloproteinases in synovium, suggesting that the development of bone and cartilage damage is a complex process influenced by multiple factors. ${ }^{30}$

Finally, this study confirms previous reports on the association of the degree of synovial macrophage infiltration with joint damage in RA. ${ }^{31}$ Accumulation of macrophages in the lining with consequent production of cytokines and matrix metalloproteinases has been implicated in erosive joint damage in RA. ${ }^{32}$ These results, together with the p53 data, lend additional support to the hypothesis that there are differential pathogenic consequences of synovitis in PsA and RA.

In conclusion this study is, to our knowledge, the first to confirm differential p53 expression in the ST of patients with RA and PsA, as well as an association of p53 expression with joint damage in RA. At the same time, our study suggests differential pathogenic consequences of synovitis in RA and PsA and points to p53 expression as a prognostic marker of joint damage in RA, underlining the need for future confirmative studies.

\section{ACKNOWLEDGEMENTS}

This work was supported by grants from the Hospital Clínic of Barcelona (Premi Fí de Residencia to GS), Societat Catalana de Reumatologia (GS), and Fundació Agustí Pedro-Pons (GS)

We thank Miguel Maestro and Jordi Ferrer of the endocrinology service of the Hospital Clinic of Barcelona for their help in p53 immunohistochemical techniques, and José Luis Pablos of the rheumatology department of the Hospital 12 de Octubre of Madrid for a cogent review of the manuscript.

\section{Authors' affiliations}

G Salvador, R Sanmarti, A Garcia-Peiró, J R Rodríguez-Cros, J MuñozGómez, J D Cañete, Unitat d'Artritis, Servei de Reumatologia, Institut $\mathrm{d}^{\prime}$ Investigacions Biomèdiques August $\mathrm{Pi}$ i Sunyer (IDIBAPS), Hospital Clinic, Villarroel 170, 08036 Barcelona, Spain

\section{REFERENCES}

1 Firestein GS, Nguyen K, Aupperle KR, Yeo M, Boyle D, Zvaifler NJ. Apoptosis in rheumatoid arthritis: p53 overexpression in rheumatoid arthritis synovium. Am J Pathol 1996;149:2143-51.

2 Firestein GS, Echeverru F, Yeo M, Zvaifler NJ, Green DR. Somatic mutations in the $\mathrm{p} 53$ suppressor gene in rheumatoid arthritis synovium. Proc Natl Acad Sci USA 1997;94:10895-900.

3 Tak PP, Smeets TJM, Boyle DL, Kraan MC, Shi Y, Zhuang S, et al. P53 overexpression in synovial tissue from patients with early and chronic rheumatoid arthritis compared with patients with reactive arthritis and osteoarthritis. Arthritis Rheum 1999;42:948-53.

4 Yamanishi Y, Boyle DL, Pinkoski MJ, Mahboubi A, Lin T, Han Z, et al. Regulation of joint destruction and inflammation by $\mathrm{p} 53$ in collagen-induced arthritis. Am J Pathol 2002; 160:123-30.

5 Gladman DD. Current concepts in psoriatic arthritis. Curr Opin Rheumatol 2002;14:361-6.

6 Ritchlin C, Haas-Smith SA, Hicks D, Cappuccio J, Osterland CK, Looney RJ. Patterns of cytokine production in psoriatic synovium. J Rheumatol 1998;25:1544-52.
7 Cañete JD, Pablos JL, Sanmarti R, Mallofré C, Marsal S, Maymó J, et al. Antiangiogenic effects of anti-tumor necrosis factor therapy with infliximab in psoriatic arthritis. Arthritis Rheum 2004;50:1636-41.

8 Danning CL, Illei GG, Hitchon C, Greer MR, Boumpas DT, Mclnnes IB. Macrophage-derived cytokine and nuclear factor $\mathrm{\kappa B}$ p 65 expression in synovial membrane and skin of patients with psoriatic arthritis. Arthritis Rheum 2000;43: 1244-56.

9 Ritchlin CT, Haas-Smith SA, Li P, Hicks DG, Schwarz EM. Mechanisms of TNF $\alpha$ and RANKL-mediated osteoclastogenesis and bone resorption in psoriatic arthritis. J Clin Invest 2003;111:821-31.

10 Hannuksela-Svahn A, Paakko P, Autio P, Reunala T, Karvonen J, Vahakangas K. Expression of p53 protein before and after PUVA treatment in psoriasis. Acta Derm Venereol 1999;79:195-9.

11 Cañete JD, Rodriguez JR, Salvador GS, Gómez A, Muñoz-Gómez J, Sanmarti R. Diagnostic usefulness of synovial vascular morphology in chronic arthritis. A systematic survey of 100 cases. Semin Arthritis Rheum 2003;32:378-87.

12 Maestro MA, Boj SF, Luco RF, Pierreux CE, Cabedo J, Servitia JM, et al. Hnf6 and Tcf2 (MODY5) are linked in a gene network operating in a precursor cell domain of the embryonic pancreas. Hum Mol Genet 2003;12:3307-14.

13 Bruynesteyn K, van der Heijde D, Boers M, Lassere M, Boonen A, Edmonds J, et al. Minimal clinically important difference in radiological progression of joint damage over 1 year in rheumatoid arthritis; preliminary results of a validation study with clinical experts. J Rheumatol 2001;28:904-10.

14 Sanmarti R, Gómez A, Ercilla G, Gratacós J, Larrosa M, Suris X, et al. Radiological progression in early rheumatoid arthritis after DMARDS: a oneyear follow-up study in a clinical setting. Rheumatology (Oxford) 2003;42:1044-9.

15 Lee CS, Portek I, Edmonds J, Kirkham B. Synovial membrane p53 protein immunoreactivity in rheumatoid arthritis patients. Ann Rheum Dis 2000;59:143-5.

16 McGonagle D, Reece RJ, Green MJ, Jack A, Veale DJ, Emery P. p53 is not demonstrable by immunohistochemistry in early rheumatoid arthritis [abstract]. Arthritis Rheum 1997;406(suppl):S119.

17 Espinoza LR, van Solingen R, Cuellar ML, Angulo J, Mendez E, Espinosa CG p53 overexpression in psoriatic skin, synovium and fibroblasts [abstract]. Arthritis Rheum 1998;41(suppl):S335.

18 Kullmann F, Judex M, Neudecker I, Lechner S, Justen HP, Green DR, et al Analysis of the p53 tumor suppressor gene in rheumatoid arthritis synovia fibroblasts. Arthritis Rheum 1999;42:1594-600.

19 Yamanishi Y, Boyle DL, Rosengren S, Green DR, Zvaifler NJ, Firestein GS Regional analysis of p53 mutations in rheumatoid arthritis synovium. Proc Natl Acad Sci USA 2002;99:10025-30.

20 Tak PP, Zvaifler NJ, Green DR, Firestein GS. Rheumatoid arthritis and p53: how oxidative stress might alter the course of inflammatory diseases. Immunol Today 2000;21:78-82.

21 Tak PP, Klapwijk MS, Broersen SFM, van de Geest DA, Overbeek M, Firestein GS. Apoptosis and p53 expression in rat adjuvant arthritis. Arthritis Res 2000;2:229-35.

22 Pap T, Aupperle KR, Gay S, Firestein GS, Gay RE. Invasiveness of synovial fibroblast is regulated by p53 in the SCID mouse in vivo model of cartilage invasion. Arthritis Rheum $2001 ; 44: 676-81$

23 Seemayer CA, Kuchen S, Neidhart M, Kuenzler P, Rihosková V, Neuman E, ef al. p53 in rheumatoid arthritis synovial fibroblasts at sites of invasion. Ann Rheum Dis 2003;62:1139-44.

24 Sun Y, Cheung HS. p53, proto-oncogene and rheumatoid arthritis. Semin Arthritis Rheum 2002;31:299-310.

25 Gladmann D, Stafford-Brady F, Chang CH, Lewandowsky K, Russell ML. Longitudinal study of clinical and radiological progression in psoriatic arthritis. J Rheumatol 1990;17:809-12.

26 McGonagle D, Conaghan PG, Emery P. Psoriatic arthritis: a unified concept twenty years on. Arthritis Rheum 1999;42:1080-6.

27 Fassbender HG. Pathology and pathobiology of rheumatic diseases, 2nd ed. Berlin: Springer, 2002:213-17.

28 Fearon U, Griosios K, Fraser A, Reece R, Emery P, Jones PF, et al. Angiopoietins, growth factors, and vascular morphology in early arthritis. $J$ Rheumatol 2003;30:260-8.

29 Reece RJ, Cañete JD, Parsons W, Emery P, Veale DJ. Distinct vascular patterns of early synovitis in psoriatic, reactive and rheumatoid arthritis. Arthritis Rheum 1999;42:1481-4.

30 Kane D, Jensen LE, Grehan S, Whitehead AS, Bresnihan B, Fitzgerald O. Quantitation of metalloproteinase gene expression in rheumatoid and psoriatic arthritis synovial tissue distal and proximal to the cartilage-pannus junction. J Rheumatol 2004;31:1274-80.

31 Mulherin D, Fitzgerald O, Bresnihan B. Synovial tissue macrophage populations and articular damage in rheumatoid arthritis. Arthritis Rheum 1996;39:115-24.

32 Chu CQ, Field M, Feldmann M, Maini RN. Localization of tumor necrosis factor alpha in synovial tissue and at the cartilage-pannus junction in patients with rheumatoid arthritis. Arthritis Rheum 1991;34:1125-32. 